\title{
One Good Turn Deserves Another: Combat versus Other Functions of Acrobatic Maneuvers in the Play Fighting of Vervet Monkeys (Chlorocebus aethiops)
}

\author{
Sergio M. Pellis, ${ }^{*}$ Vivien C. Pellis, ${ }^{1}$ Louise Barrett, ${ }^{1,2}$ and S. Peter Henzi ${ }^{1,2}$ \\ ${ }^{1}$ University of Lethbridge \\ ${ }^{2}$ University of South Africa \\ *Corresponding author (Email: pellis@uleth.ca)
}

Citation - Pellis, S. M., Pellis, V. C., Barrett, L., \& Henzi, S. P. (2014). One good turn deserves another: Combat versus other functions of acrobatic maneuvers in the play fighting of vervet monkeys (Chlorocebus aethiops). Animal Behavior and Cognition, 1(2), 128-143. doi: 10.12966/abc.05.04.2014

\begin{abstract}
During play fighting, animals make a variety of movements, some of which seem unrelated to the ongoing actions of the partner. Among such movements are the jumps and rotations reported in many species of Old World monkeys. In the present study, videotaped sequences of jumps and rotations performed by juvenile vervet monkeys were analyzed. Using the Eshkol-Wachman Movement Notation (EWMN) the sequences were described frame-by-frame revealing that about $82 \%$ of the jumps and rotations were correlated with the movements performed by the partner, consistent with the view that these movements are used as tactics of attack and defense. The majority of the remaining $18 \%$ occurred in contexts in which the performer solicited playful attacks from the partner. A small number of the rest were performed in a manner consistent with the movements being imposed by the performer to increase the difficulty in contacting the partner. The same distribution was present in both captive and free-living monkeys. Thus the analyses show that while most jumps were combat-related, these movements can occur in a variety of functional contexts.
\end{abstract}

Keywords - Play fighting, Vervet monkeys, Eshkol-Wachman movement notation, Acrobatic maneuvers, Combat, Play solicitation

Play fighting involves competition over gaining some advantage, with that advantage in primates, and for many other mammals, often involving gaining access to some species-typical body target, which may be bitten, nibbled or nuzzled if successfully contacted (Aldis, 1975; Biben, 1998; Pellis, 1988; Symons, 1978). Maneuvers performed during play fighting, just as in the case of serious fighting (Blanchard \& Blanchard, 1994; Geist, 1978; Pellis, 1997), may be viewed as tactics of attack and defense (Pellis \& Pellis, 1998), with species differences arising from the species-typical agonistic repertoire and the maneuverability capability of each species (Meaney, Stewart, \& Beatty, 1985). However, although most behavior patterns used in play are derived from species-typical functional contexts (e.g., aggression, courtship, predation) (Heymer, 1977; Millar, 1981), some behavior patterns are unique to the play context (Petrů, Śpinka, Charvátová, \& Lhota, 2009). For example, during play, juvenile monkeys and apes of several species may engage in leaps and rotations that are not apparent in non-play contexts (Nishida \& Inaba, 2009; Pellis \& Pellis, 2009). At least five functional hypotheses have been proposed that could account for these acrobatic actions during play fighting.

First, acrobatics may be inserted into playful sequences to promote physical training (Brownlee, 1954; Simpson, 1976), with species differences in the amount and complexity of such acrobatics incorporated into play reflecting species differences in maneuverability (Fontaine, 1994). Second, they may occur as a means of training for the unexpected (Špinka, Newberry, \& Bekoff, 2001), with the leaps 
and rotations being inserted to make task completion more difficult and so provide the juvenile with experiences that teach it to take unexpected disturbances in their stride (Petrů, Špinka, Lhota, \& Š́pek, 2008). Third, the acrobatics performed by one player may be used to induce play in another and so function as play signals (Palagi, 2008). Fourth, acrobatic actions may be used as tactics to evade or facilitate playful attack and defense (Pellis \& Pellis, 1998). Another hypothesis, that of using acrobatic actions as a sexually selected behavior to gain favor with potential future mates, was beyond the capacity of present study to be tested. In any case, where tested, this hypothesis has not been supported (Nishida \& Inaba, 2009).

The four hypotheses tested in this paper may not be mutually exclusive, with different types of acrobatic maneuvers being accounted for by different hypotheses. For example, in rats, some of the leaps performed are used as tactics to overcome the partner's defenses (e.g., forward leap), whereas some occur independently of the movements of the partner (e.g., upward hop) (Pellis \& Pellis, 1983). The former would thus be explained by the combat hypothesis, whereas the latter is more consistent with the motor training, training for the unexpected or signaling hypotheses. Therefore, acrobatic maneuvers during play fighting may serve different functions. Given that acrobatic actions during the play of any particular species may be consistent with multiple hypotheses, it cannot be assumed a priori why any one particular acrobatic action is performed.

The combat hypothesis differs from the others in a crucial manner. If these maneuvers serve as combat tactics, then all leaps and rotations would occur in the context of gaining or avoiding contact with the species-typical body target. The other three hypotheses would be united in predicting the opposite, albeit with likely subtle differences in context. For example, to serve a communicatory function, the acrobatics of the performer would occur prior to a playful attack or when a potential partner is beginning to turn away and so disengaging from play. In contrast, the physical training hypothesis would predict that such acrobatics would likely occur when initiating play or when disengaging from play, moments in the interaction that offer the greatest opportunity to perform acrobatics with maximum exertion, and so motor training. The training for the unexpected hypothesis would predict acrobatic movements at all stages of the encounter, but especially at times when the performer gains the advantage, which would increase the likelihood of losing control of the encounter to the other animal and so increase the experience of unexpected events. Such sabotaging of an advantage has been demonstrated to occur in juvenile rats when playing (Foroud \& Pellis, 2002, 2003), leading to an increased likelihood of a role reversal (Pellis, Pellis, \& Foroud, 2005). The core difference in predictions is that the combat hypothesis predicts a tight correlation between the acrobatic maneuvers of one partner with the combat maneuvers of the other, whereas the other hypotheses predict no such correlation.

To test these divergent predictions, videotaped sequences of play fighting in vervet monkeys (Chlorocebus aethiops) were analyzed using the Eshkol-Wachman Movement Notation System (EWMN). This system can be used to track the movements of each animal relative those of the partner (Golani, 1976; Pellis, 1989), allowing leaps and rotations that are not correlated with the movements of the partner to be distinguished from those that are (see Methods for details).

\section{Method}

\section{Subjects}

Juvenile monkeys from a troop of captive vervet monkeys (Chlorocebus aethiops) housed at the Werribee Open Range Zoo (near Melbourne, Australia) were observed in August 2001. They were housed in a large, outdoor enclosure with grassed areas, surrounded by trees and bushy patches. From the public viewing area, most of the enclosure was visible through a glass wall. There were 4 juveniles ( 3 males, 1 female) that ranged between 1-3 years of age. In many of the play fights, the youngest and oldest juveniles, both males, were individually distinguishable, but the two in-between juveniles were not distinguishable from each other in most cases, but they were from the other two. So, where possible, when they played together, data were scored for the two individually identifiable males and for the two 
intermediate juveniles as a composite. For some measures (see below), this breakdown was used to verify that any emerging pattern was not due to the actions of one, idiosyncratic animal. While play fighting may differ in frequency and roughness with age and sex (Biben, 1998; Fagen, 1981), the same targets and tactics are used across these conditions (e.g., Pellis \& Pellis, 1997; Pellis, Pellis, Reinhart, \& Thierry, 2011; Reinhart, Pellis, Thierry, Gauthier et al., 2010; Symons, 1978). In the present study, when all four juveniles were playing in the central grassy area, and so all simultaneously visible, it was apparent that all of them attacked and defended the same body targets and performed the same range of acrobatic maneuvers.

Given that there can be significant intra-specific variation in behavior across troops of the same species (Thierry et al., 2008; de Waal \& Luttrell, 1989), and in particular, that novel behaviors have been reported to arise in captivity (Laidre, 2008), caution needs to be exercised in drawing conclusions about a species from the behavior of one troop of captive animals. Confirmation of the pattern of behavior in other troops, especially free-living ones, is important (Pellis et al., 2011; Petit, Bertrand, \& Thierry, 2008; Reinhart et al., 2010). Therefore, to verify that the body target competed over during play fighting and the pattern of use of acrobatic movements in the juveniles from the Werribee troop is typical of vervets in general, we also videotaped and analyzed the play fighting of wild juvenile vervet monkeys. These juveniles were members of three habituated study groups $\left(\mathrm{N}_{1}=\sim 30\right.$ animals, $\mathrm{N}_{2}=\sim 50$ animals, $\mathrm{N}_{3}=\sim 70$ animals) at the Samara Game Reserve in Eastern Cape Province, South Africa (Pasternak et al., 2013).

Over the course of 4 days, the juveniles from the Werribee troop were videotaped, opportunistically, using a Sony $8 \mathrm{~mm}$ Camcorder. The tapes were then converted to VHS format, with a time code $\left(1 / 30^{\text {th }}\right.$ of a second) being added during dubbing using a Horita TRG-50 time encoder (Horita, Mission Viejo, CA). The $407 \mathrm{~min}$ of videotape contained 535 play fights.

The wild troops in South Africa have been under constant observation since 2008. Data for these analyses were collected over six months in 2009 and six months in 2013 as part of a larger program of behavioral research. During all-day follows of the troops, bouts of play fighting were recorded ad libitum using either a Sony 8mm Camcorder or a Canon Powershot SX50 digital camera. Tapes from the camcorder were digitized, and files from the digital camera downloaded as .MOV files, for subsequent analysis.

From the recorded sequences, it was possible to identify animals between 1-3 years of age, but tracking individuals and differentiating reliably between the sexes was not possible. For present purposes, of the hundreds of play fights recorded, the first 100 play fights in which the beginning and end of contact was observable were used.

\section{Behavioral analyses}

In many Old World monkeys, rough-and-tumble play involves both chasing and wrestling (Owens, 1975a; Reinhart et al., 2010; Symons, 1978), but for the current study, only interactions in which there was an attempted bite or grab (i.e., contact) were considered to constitute play fighting. Furthermore, only acrobatic maneuvers occurring in the context of play fighting were analyzed. Play fighting involves competition over gaining some advantageous contact and so can be potentially confused with serious fighting (Aldis, 1975; Pellis \& Pellis, 1998). However, several criteria can be used to distinguish playful from serious fights (Smith, 1997). In playful fights, (1) a resource, such as a piece of food, is not gained or protected, (2) the contact is restrained, or, at least, there are no combat-induced injuries, (3) there are frequent role reversals between the pair mate that is the attacker and the one that is the defender, (4) even if chasing ensues following contact, further affiliation is likely, and (5) the open mouth play face is typically associated with the contact. Only interactions meeting these criteria were considered to constitute play fighting and were used for further analysis.

Two main analyses were conducted. As most animals contact a particular body target during play fighting (e.g., Aldis, 1975; Pellis, 1988), which in most primates involves biting, albeit restrained (e.g., Biben, 1998; Owens, 1975a; Pellis \& Pellis, 1997; Symons, 1978), the body areas bitten were scored. To ascertain the targeted body area, the first initiating bite in a play fight was recorded, as was the first 
retaliatory bite by the partner. Once in a wrestle, biting can be more indiscriminate (Aldis, 1975). The initiating bite and the first retaliatory bite provide evidence of the body target that is competed over during play fighting. Following the method used in previous comparative studies of primates, the vervet's body was divided, hypothetically, into 5 areas (Pellis \& Pellis, 1997; Reinhart et al., 2010): (1) Head and face, including the top, front and side of the head, (2) neck, shoulders and upper arms, with the arm from the elbow upwards included, (3) body, which includes the dorsum and lateral areas posterior to the shoulders and the ventrum, (4) lower arms and hands, which includes the arm from below the elbow, and (5) legs and feet.

From the first videotape from Werribee (based on 215 play fights), in 124 cases in which the beginning of the encounter was observable, the first bite delivered by the approaching monkey was scored (offensive bites). Of these, 93 involved the monkey that received the bite retaliating with a bite of its own (defensive bites). Using the sequences from the second videotape, the first 20 offensive bites and the first 20 defensive bites were scored for the oldest and the youngest males. In addition, the first 20 offensive bites and the first 20 defensive bites were scored for the two other juveniles, but these were pooled together as their individual identity could not be tracked consistently (see above). For all bites, the context was recorded. This included whether the animals were standing or sitting and whether they were in an unconstrained context (i.e., away from obstructing objects, such as walls and trees) and on stable substrate (i.e., on flat terrain as opposed to balancing on tree branches).

Video clips containing sections of play fights with acrobatic movements were selected from the first videotape. The first 30 in which the movements of both animals could be clearly observed (i.e., not obscured by trees or other monkeys) and in which the monkeys occupied at least $1 / 3$ of the video screen were used. To qualify for inclusion, the acrobatic maneuver had to include a jump (forward, sideways, backwards or straight up) and a rotation, which can involve any one of three axes - roll: around the longitudinal axis of the body; pitch: around the axis running through the middle of the body, from left to right; yaw: around a vertical axis running at $90^{\circ}$ through the body, from dorsum to ventrum. These sequences were analyzed frame-by-frame using the Eshkol-Wachman Movement Notation (EWMN) (Eshkol \& Wachman, 1958).

In brief, EWMN is a globographic system, designed to express relations and changes of relation between parts of the body, with the body treated as a system of articulated axes (i.e., body and limb segments). A limb is any part of a body that either lies between two joints or has a joint and an extremity. These are imagined as straight lines (axes), of constant length, which move with one end fixed to the center of a sphere. The body is represented on a horizontally ruled page into columns that denote units of time (e.g., frames of a video). The signs for movement are read from left to right and from bottom to top. Movements by any limb segment, or the body as a whole, can be described as the distal end moving across the surface of the sphere, with the proximal end being anchored in the center of the sphere. Typically, the locations on the sphere (horizontal and vertical) are at $45^{\circ}$ angles, but the unit of angular measurement can be reduced (e.g., 22.5 ) if finer grain comparisons are needed. An important feature of EWMN is that the same movements can be notated in several polar coordinate systems. The coordinates of each system are determined with reference to the environment, to the midline axis of the subject's body, and to the next proximal or distal limb or body segment. Critical for studying interactions between animals, the movement by one animal can be described as relative to the body of the other animal. By transforming the description of the same behavior from one coordinate system to the next, invariance in the behavior may emerge in some coordinates but not others. Thus, the behavior may be invariant in relation to some or all of the following - the subject's longitudinal axis, gravity, bodywise in relation to the next proximal or distal segment (e.g., Eilam \& Golani, 1988; Golani, 1976; Pellis, 2011), or to some aspect of the opponent's body (e.g., Moran, Fentress, \& Golani, 1981; Pellis, 1982).

Critically, for this study, the movements of the two animals were assessed so as to determine whether they were correlated with one another. To do this, three measures of inter-animal relationship were tracked over frames: the distance between monkeys (in monkey lengths, excluding the tail), the angular orientation of the longitudinal axis of the bodies and the parts of their bodies that were in closest opposition or in contact. When combined with the movements of the bodies and heads of the two 
monkeys, these inter-animal measures could be used to determine whether some aspect of the interanimal relationship was kept constant. That is, what could be distinguished using this analytical framework was whether, despite the movements made by both animals, an inter-animal relationship was kept constant, or, if inter-animal relationships varied independently of the animals' movements (see Pellis et al., 2013, especially Appendix A for a detailed description of how this system is used and read). For the present paper, drawings of real or abstracted monkeys will be used to represent the patterns of behavior extracted from the EWMN scores.

Finally, an additional 30 sequences containing acrobatic maneuvers were selected from the second videotape, using the same criteria as above. These were inspected frame-by-frame to verify the patterns identified in those analyzed using EWMN. In addition, the context in which the acrobatic movements were used was recorded. This included determining whether the movements facilitated attack, defense or neither. If the latter was the case, then what ensured immediately after, such as an attack by the partner, was also recorded. Additional sampling of the videotaped material and measurements that were used to test hypotheses as they arose in the analyses are described as needed in the Results.

\section{Confirming the pattern}

The first 60 offensive bites and associated defensive bites that could be ascertained in the South African sample were scored with regard to the body area bitten and the context in which the monkeys launched the attacks. Then, 50 sequences from South Africa that contained acrobatic movements were analyzed frame-by-frame to determine whether they were combat-related or not, and, if so, how they facilitated combat.

Statistical analyses

Given that most of the data were categorical, statistical comparisons involved Chi-squares (Siegel \& Castellan, 1988). To verify that the behavior recorded by the primary scorer (SMP) was recognizable by another observer, a second individual (VCP) re-scored 30 offensive and 30 defensive bites and 30 sequences involving acrobatic movements. The inter-observer reliability between scorers was high (correlation coefficients $\geq 0.90$ ).

\section{The Werribee juveniles}

\section{Results}

Targets. The pooled data for bites derived from the large sample showed that neither offensive nor defensive bites were randomly distributed (Figure 1). Assuming a null hypothesis that bites to all 5 areas of the body were equally likely showed that there was a significant difference (offensive bites: $X^{2}=$ 268.35, $d f=4, p<0.001$; defensive bites: $X^{2}=158.34, d f=4, p<0.001$ ). The offensive bites were disproportionately directed at the neck, shoulder and upper arm area and the defensive bites were disproportionately directed at the head. The data for the two individual males and the composite of the other two juveniles showed the same modal targets for both offensive bites (neck, shoulder and upper arm area: $75 \%, 70 \%, 60 \%$, respectively) and defensive bites (head: $75 \%, 65 \%, 80 \%$, respectively). In each case, the modal target was bitten more frequently than that expected by chance (Chi-square analyses, $p<$ $0.05)$.

In scoring bites, it became evident that the monkeys were less likely to perform acrobatic movements in some substrates. For example, when approaching one another, face-to-face on a horizontally inclined $\log$, the monkeys grabbed one another when within about half a body length, then, as they sat on their haunches, pulled each other into close, front-to-front contact. From this position, one monkey would launch a bite to the upper arm, shoulder or side of the neck of another monkey, who would then retaliate with a bite to the side of the attacker's face. Acrobatic movements did not occur in these cases or in similar situations in which the animals' movements appeared to be constrained, such as when the monkey being approached was pressed against a wall, rock or in dense vegetation (Figure 2). 
Pellis et al. 133

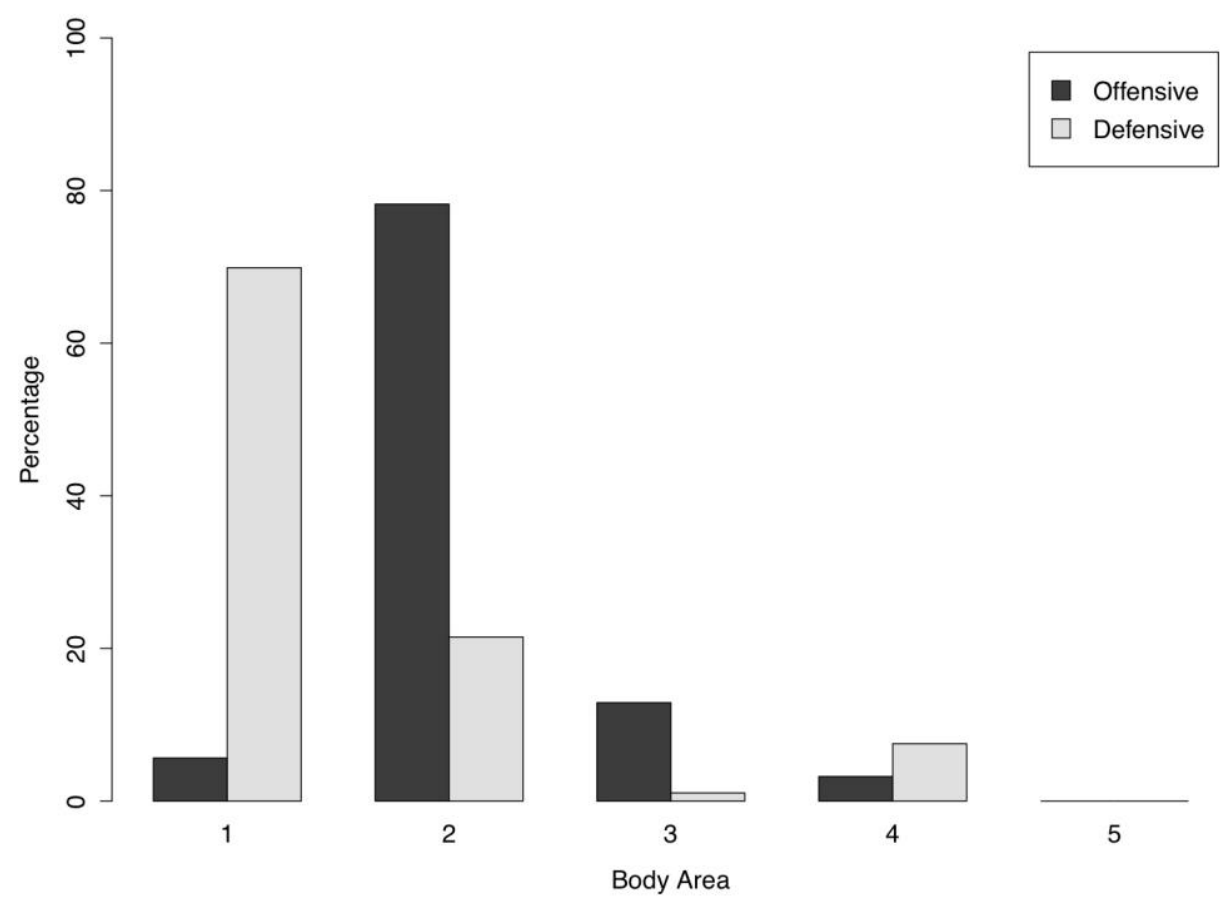

Figure 1. The distribution of initiating (offensive) bites and the distribution of retaliatory (defensive) bites are shown over the different areas of the body. The body areas are: (1) head, (2) neck, shoulder and upper arm, (3) the remainder of the torso, (4) lower arms and hands and (5) legs and feet.

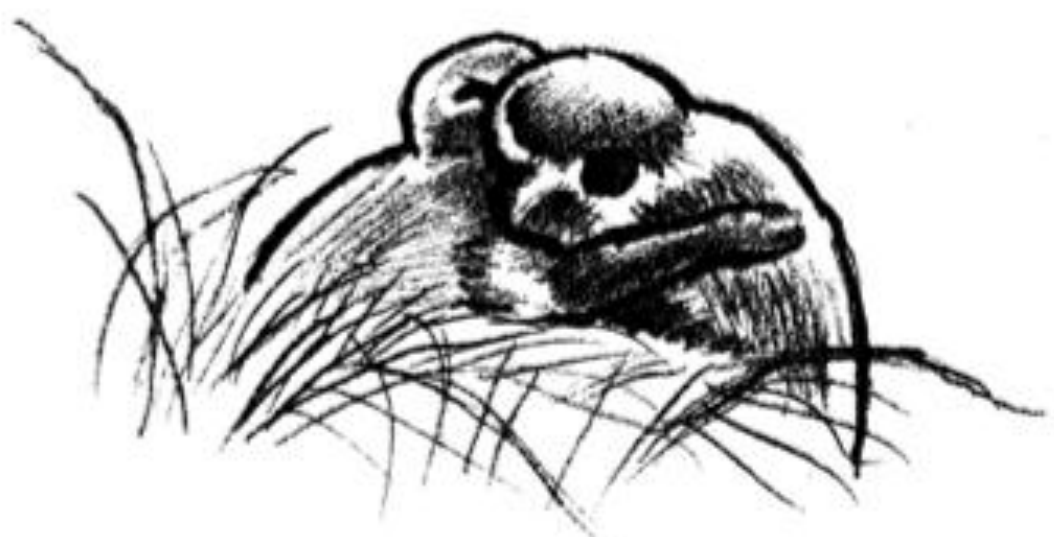

Figure 2. Two monkeys are in a frontal embrace while nestled in tall clumps of grass, with the monkey on the right directing a bite at the partner's upper arm. Drawing taken from a video frame.

To test this impression quantitatively, the first 20 bites in which the monkeys were constrained by sitting next to one another on a log, on branches or pressed against an obstacle (e.g., wall, rock) were scored as well as the first 20 bites that occurred when one monkey approached another in an open area for whether the interaction included acrobatic maneuvers. These were collected from the videotape following scoring the offensive bites above ended (i.e., these samples of bites were independent of one another). Only $5 \%$ of the constrained attacks contained an acrobatic action, whereas $85 \%$ of the unconstrained cases did so, a significant difference $\left(X^{2}=25.86, d f=1, p<0.001\right)$. Most of the unconstrained attacks occurred in the large, grassed area in the center of the outdoor enclosure. 
Acrobatics. EWMN analysis of the 30 cases of acrobatic movements revealed that the majority $(86.7 \%)$ involved correlated actions between the attacker and the defender in the context of combat $\left(X^{2}=\right.$ 16.1, $d f=1, p<0.01)$. For example, in Figure 3, an attacker approaches from the left (a), and, when it comes to within half a body length of the other juvenile, it leaps forward, directing its mouth to its partner's left shoulder. As it comes close to contact, its partner turns to face and the attacker jumps upwards and rotates its body, pivoting around a vertical axis that runs through its head, and, while it does so, looks down at its partner. Meanwhile, the partner tracks the attacker's movements, keeping a face-toface orientation (b). The attacker continues to move around its partner, who similarly continues to track its attacker's face (c). Then, as the attacker turns its head to look down in the direction to which it is falling, its partner crouches and directs its face to the exposed side of the attacker's head and shoulder (d), but as the partner lunges forward, the original attacker lands and turns to face its partner, blocking the attack (e).
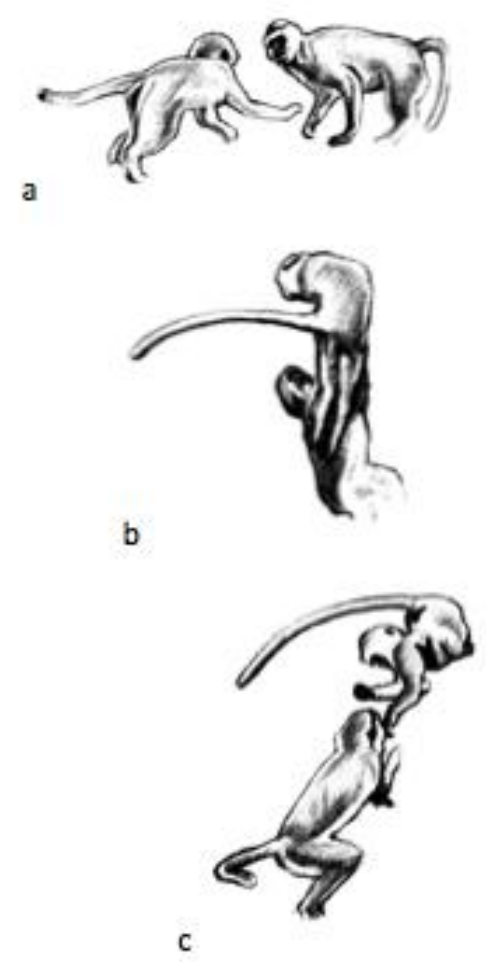
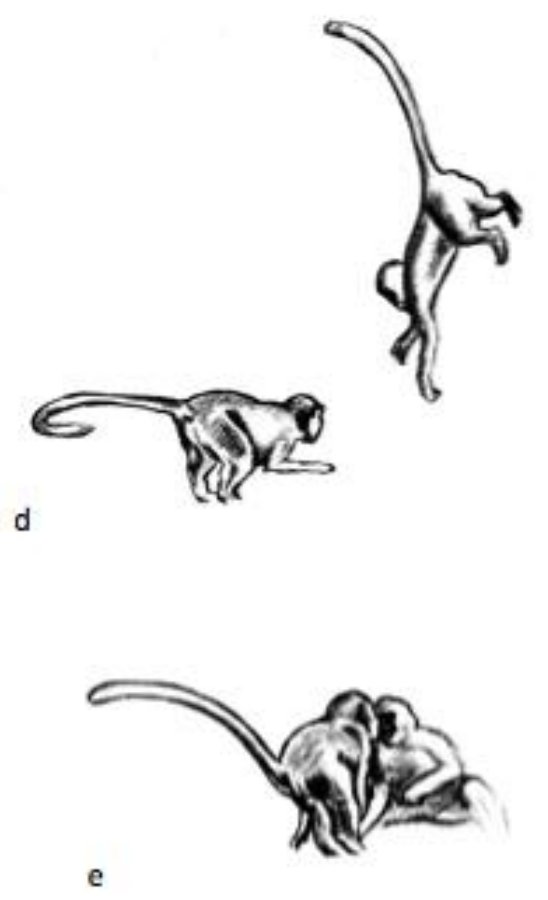

Figure 3. The sequence shows a sequence of jumps and rotations as the monkey on the left attacks the monkey on the right (see text for details). The drawing is based on a videotaped sequence.

In this example, the attacker incorporates a jump to the side and upwards, coupled with a rotation of the longitudinal axis of its body around a vertical axis, pivoting around its nose, to overcome its partner's blocking action of turning to face. That is, the jump and rotation arise as a combat maneuver so as to overcome the defensive action of the defender. Most of the other jumps and rotations were similarly used to attack, defend or counterattack. The non-combat related jumps and rotations were not correlated to the movements of the partner. Rather, they involved jumping and rotating, around one or more axes, either away from the partner or parallel to the partner. That is, the movements of the performer were independent of those of the partner and did not contribute to either attack or defense.

Characterizing combat related acrobatics. Given that the EWMN analysis indicated that most acrobatic maneuvers begin as a combat tactic by the attacker, but then, because of the defensive actions of the partner, the maneuvers by the attacker become more complex, combining aspects of attack and 
defense (see Figure 3), a different sample of attacks that led to incorporating acrobatic movements was analyzed. Sampling from a previously unviewed section of videotaped material, the first 60 instances of acrobatic movements involving the attacker were viewed frame-by-frame. When comparing the axis of rotation and the combination of movements across the acrobatic maneuvers, several distinct forms were characterized.

When the approaching monkey attacked in an unconstrained setting, it typically maneuvered to attack the target area while avoiding the defender's mouth. In order to do this, the jumps and rotations were integrated in several ways.

(i) The attacker approaches, directly, from the front, to between 0.5-1 body lengths from its partner, crouches and then leaps forward, rotating cephalocaudally around its longitudinal axis as it does so. The attacker lands on its back, with its face pointing up at the upper arm or throat of its partner, and, from this position, lunges up to bite. The defender can block this attack by jumping backwards or upwards.

(ii) The attacker approaches, directly, from the front, to between $0.5-1$ body lengths from its partner, then jumps forward and rotates around its vertical axis, keeping its ventrum horizontal to the ground, pivoting around its head, so that as it lands, it is oriented perpendicular to the defender's neck area (Figure 4A). The defender can block this attack by turning to face the attacker's face, but which, in turn, can lead to further lateral movement by the attacker as it maneuvers to access the defender's shoulder (Figure 4B). Continued movement and countermovement can lead to a reversal of the animals' original starting positions (Figure 4C).

(iii) The attacker approaches, directly, from the front, to between 0.5-1 body lengths from its partner, then jumps forward and rotates around its vertical axis, but also rotates, so that the longitudinal axis of its body is held vertically, with its head facing downwards. The monkey's body pivots around its head with its face opposing the shoulder that is bitten if the defender does not respond. As in the previous case (ii), the defender can block this by turning to face its attacker and jumping, moving its body away from its attacker.

A.

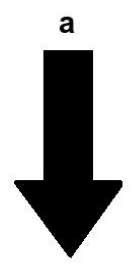

B.

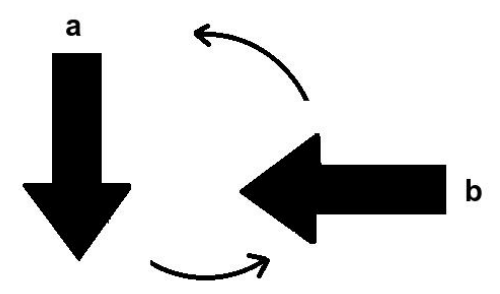

c.
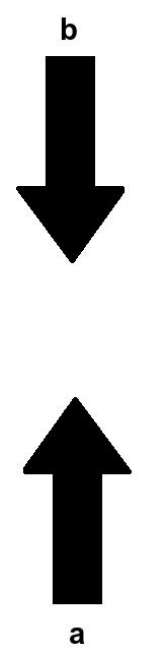

Figure 4. The schematic drawing shows a dorsal view of two monkeys (thick arrows) as b attacks a. In panel $\mathbf{A}, \mathrm{b}$ jumps and rotates to a's left facing a's shoulder area. Then in panel $\mathbf{B}$, a rotates to face b, blocking the attack, but this then leads $b$ to jump and rotate further anti-clockwise as a continues to track, so that in panel $\mathbf{C}$, a and b have reversed their original starting positions. 
(iv) The attacker approaches, as in the cases described above, but once a jump towards its partner begins, the movements performed are difficult to characterize as they contain elements that seem to be a mixture of the ones above.

Characterizing non-combat related acrobatics. Again, from the videotaped material not previously sampled, 30 acrobatic movements that occurred in close proximity to other monkeys ( $\leq 2$ body lengths) were inspected frame-by-frame. The axes around which these rotations occurred and the consequences of those movements (e.g., subsequent action by the performer or a nearby partner) were recorded. Three patterns were characterized.

(i) The monkey could jump sideways or backwards away from its nearest partner and these jumps could be combined with a rotation. When jumping forward, the monkey rotated its head around an axis running laterally through the flanks of its body, landing on its back (i.e., a somersault). When jumping sideways, the rotation involves a different axis. Whatever the rotations involved, the performer does not attempt to grab or bite the partner, but the partner was likely to attack the nearby partner (see below). Such an apparent solicitation leading to an attack by the partner is illustrated in Figure 5. The monkeys are a little more than 2 body lengths apart, with the animal in the front sitting perpendicular to the approaching partner, and facing the right (a). From this position, the monkey at the rear jumps forward and as it closes to about a third of a body length away in front of the sitting partner, it summersaults over its own head (b), so that when it lands, it is lying on its back, in front of its partner, which lowers its head to face the supine monkey (c). The sitting partner then rose up and lunged to attack the supine monkey (d).

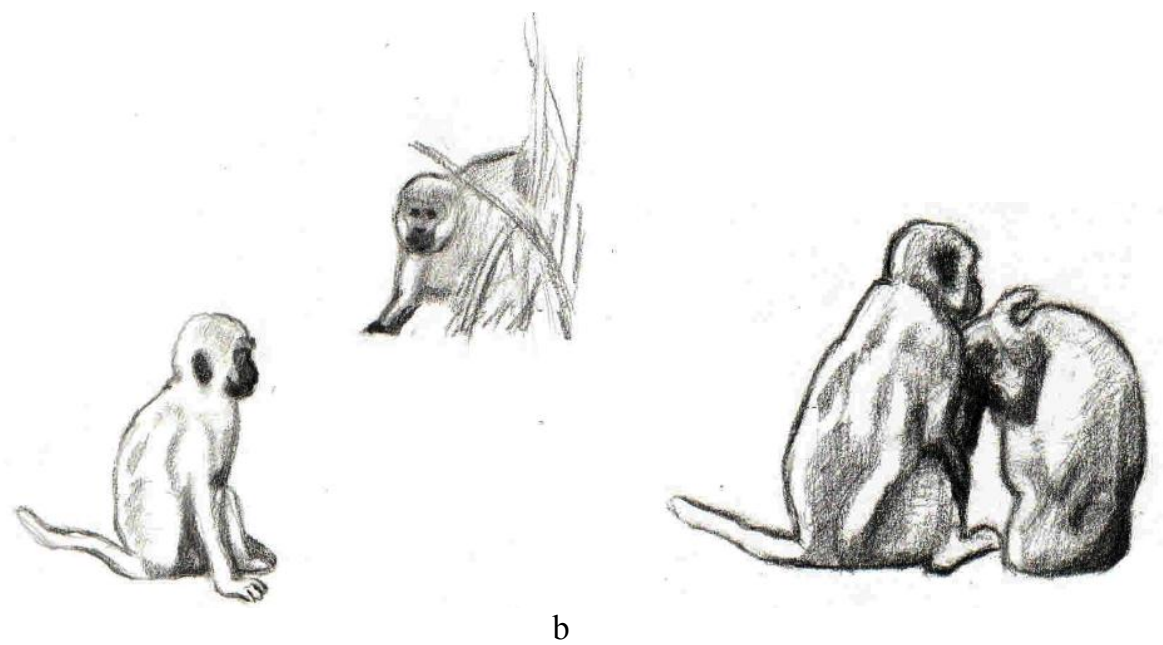

a

b

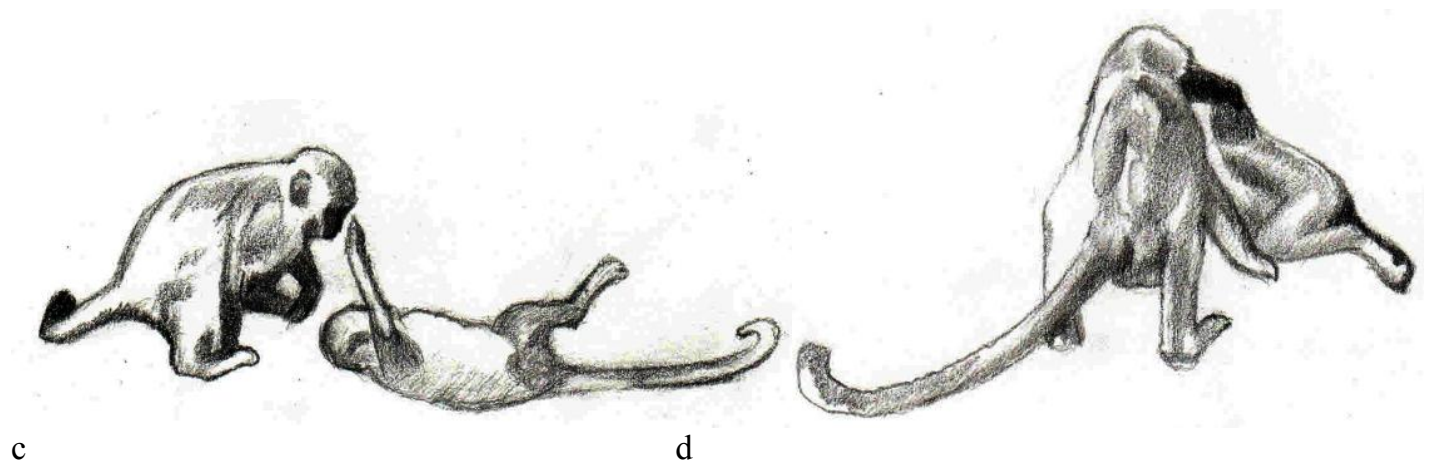

Figure 5. The sequence shows the furthest monkey leaping and then rotating to land on its back in front of the partner, who then lunges forward and attacks. Drawn from a videotaped sequence. 
(ii) The attacker approaches, directly, from the front, to between 0.5-1 body lengths from its partner, then jumps forward and rotates around a vertical axis, but also rotates so that the longitudinal axis of its body is held vertically, with its head facing downwards. The monkey's body pivots around its midbody, with its ventrum facing the shoulder area of its partner; the head and the rump both traverse a large arc. In these circumstances, while still in the air, the performer could grab at its partner with an open mouth, suggesting an attack of sorts. However, given the monkey's precarious position in the air, the performer does not typically succeed in making contact.

(iii) One monkey approaches or is near another, then performs a combination of jumps and rotations that are difficult to characterize in that they contain elements that seem to be a mixture of the ones above.

Of the 30 cases, $56.7 \%$ led to the monkey becoming engaged in a play fight, and for the longitudinal axis rotation, this was evenly split between being attacked and the performer itself attacking a nearby partner (60\% versus $40 \%$ ). None of the other forms of acrobatic maneuver ended with the performer attacking a nearby monkey, although this may be partly due to the lower frequency of these movements (see below). Two of the longitudinal axis rotations involved the performer rolling in front of its partner in a perpendicular orientation and these were coupled with unsuccessful grabbing attempts toward its partner. Similarly, $60 \%$ of the horizontal rotations combined with vertical axis rotation involved unsuccessful grabbing attempts toward one's partner.

In both combat and non-combat situations, the different types of acrobatic maneuvers occurred at differing frequencies (Table 1), with some being significantly more frequent than others (combat: $X^{2}=$ 13.70, $d f=3, p<0.01$; non-combat: $X^{2}=13.46, d f=3, p<0.01$ ). Given that individuals could not be reliably tracked across instances, it is possible that some individuals performed some of the maneuvers more frequently. Nonetheless, in situations in which all 4 juveniles were playing in the open field simultaneously, it was clear that all individuals could perform all the maneuvers.

Table 1

Frequency of Different Types of Acrobatic Movements in Combat $(n=60)$ and Non-combat $(n=30)$ Contexts

Combined

Horizontal/vertical

\begin{tabular}{|c|c|c|c|c|}
\hline & Long axis rotation & Horizontal rotation & $\begin{array}{l}\text { rotation } \\
\text { rof }\end{array}$ & Other \\
\hline Combat & $31.7 \%$ & $40.0 \%$ & $16.7 \%$ & $11.6 \%$ \\
\hline Non-Combat & $53.3 \%$ & $20.0 \%$ & $16.7 \%$ & $10.0 \%$ \\
\hline
\end{tabular}

\section{The South African juveniles}

The first 60 offensive bites and the 44 defensive (retaliatory) bites associated with those offensive bites were scored for the South African troops. For the offensive bites, the modal target area was the side of the neck, shoulder and upper arm (76.7\%) and most of the defensive bites were directed at the side of the face and head (77.3\%). Comparing across the five target areas, the distribution of bites for both offense and defense was not random $\left(X^{2}=120.82, d f=4, p<0.001\right.$ and $X^{2}=83.55 ; d f=4, p<0.001$, respectively). Moreover, the difference in the distribution of offensive and defensive bites was significant $\left(X^{2}=728.6, d f=4, p<0.001\right.$, in which a goodness of fit for defensive bites was based on the percent distribution of offensive bites). Finally, splitting the bites scored in 2009 and 2012, and analyzing them separately showed the same, non-random distributions as when the data from these two years were lumped together $(p<0.05)$. Therefore, the targeting of bites during play fights in the free-living animals was the same as that in the captive troop.

With regard to acrobatic maneuvers, the first 50 play fights, in which such an action was performed, was scored. Of these acrobatic maneuvers, $78 \%$ were associated with combat, used either to gain or evade a bite. This was a significantly non-random distribution $\left(X^{2}=15.68, d f=1, p<0.001\right)$. For 
both years, the modal pattern was the same, with most acrobatic maneuvers being performed in a combatrelated context (2009: 73.2\% 41 cases; 2012: 100\% of 9 cases). Moreover, most of the non-combat acrobatic maneuvers involved moving in a way that was consistent with soliciting attack from the nearest partner (81.8\%). As for the Werribee troop, acrobatic maneuvers were more likely to occur when the animals were playing in an open space. Therefore, with regard to acrobatic maneuvers, the free-living troops showed the same pattern as that in the captive troop.

\section{Discussion}

As is typical of many Old World monkeys (e.g., Owens, 1975a; Pellis \& Pellis, 1997; Reinhart et al., 2010; Symons, 1978) and apes (Schaller, 1963), in vervets, the body target competed over during play fighting is the shoulder area (extending to the side of the neck and down the upper arm). Defensive bites are mostly directed at the head, especially, the side of the face, again, similar to what has been found in other Old World monkeys (Owens, 1975a; Pellis \& Pellis, 1997; Reinhart et al., 2010; Symons, 1978). Based on the scars recorded - presumably arising from intra-specific fighting - the head, neck and shoulder area is the area that is most often targeted in the serious fighting of many species of Old World monkeys (e.g., Hausfater, 1972; Ruehlmann, Bernstein, Gordon, \& Balcaen, 1988; Whitten \& Smith, 1984), suggesting that juvenile play fighting in species like vervets is a simulation of intra-specific fighting. This conclusion is supported by a study that explicitly compares serious and playful fighting in baboons (Owens, 1975b). The acrobatic maneuvers of the vervet monkeys were examined within the context that, during play fighting, they attack and defend the shoulder area and need to overcome the risk of retaliatory bites to the side of the face. It should be noted that these same targets were observed in the captive troop and in the free-living troop, supporting the likelihood that this organization of play fighting is species-typical.

The acrobatic maneuvers performed by vervet monkeys during play fighting can be spectacular, sometimes combining high leaps with rotations around all three axes (roll, pitch and yaw). The findings from this study show that most of these acrobatic maneuvers were performed in conjunction with attack and defense, with the added complexity arising from the moves, countermoves and counter-countermoves between the two partners (see Figure 3). That is, about $82 \%$ (averaging across the captive and wild troops) of all such acrobatic maneuvers served as tactics of attack and defense. Therefore, of the competing hypotheses posited to account for the presence of acrobatic maneuvers in the play fighting of vervets, the combat hypothesis is the one that is the most supported, as most acrobatic maneuvers function to facilitate gaining access to, or blocking access to, the play target. Again, it should be noted that as the same pattern was found both in the captive and free-living troops, the possibility that this conclusion likely applies to vervet monkeys in general and is not an artifact of one aberrant individual or troop is supported.

\section{A methodological caveat}

In the captive troop, a formal analysis of the role of substrate in the likelihood of performing acrobatic maneuvers showed that acrobatic movements were more likely to be used in the unconstrained contexts; for example, when in the middle of an open field with short grass, rather than when playing on tree branches or where the animals were pressed against clumps of tall grasses or bushes. Casual observation of the free-living troops conveyed the same impression. This has also been found for serious fighting in rodents, with most studies reporting staged encounters in relatively small enclosures, usually of a square or rectangular shape (e.g., Blanchard, Blanchard, Takahashi, \& Kelley, 1977; Pellis \& Pellis, 1992). In such settings, the defending animal often adopts a standing posture with its back pressed against one of the corners, severely limiting the tactics that can be deployed by the attacking animal (Pellis, 1989). In such situations, it is only by scoring those cases in which the attacker has an unfettered opportunity to attack, such as when the defender is near the center of the cage, that species differences in preferred attack tactics become evident (Pellis, Pellis, Pierce, \& Dewsbury, 1992). This consideration is 
critical when comparisons of acrobatic movements during play are made across species of monkeys. For example, Hanuman langurs have been reported to make a variety of rotational movements during both social and solitary play. It seems likely that, from the pattern of their performance, that this species is less likely to use rotational movements for combat, and more likely to do so as training for the unexpected (Petrů et al., 2008). To compare fairly across species, however, it is not the overall frequency of performance that needs to be compared, but what the subjects do when they have an unconstrained opportunity to perform such movements. Such data can then be used to assess whether one species performs such movements for one functional reason and whether another species does so for another functional reason. If the opportunity for performance is not taken into account, misleading conclusions may follow.

\section{Dominant and subsidiary functions}

Even though the combat hypothesis accounted for about $82 \%$ of the acrobatic maneuvers performed by the vervets, this left about $18 \%$ that were performed in a manner that did not facilitate combat. Of these, $10 \%$ were indeterminate as to what function they may serve, but the others are linked to one of two contexts that are consistent with two of the other posited hypotheses (from data shown in Table 1). Most of the rotations around the longitudinal axis (roll) (87.5\%) and all those around the lateral axis (pitch) seemed to be performed in ways that are consistent with what have been described as selfhandicapping actions that function to solicit attack from a partner (Bekoff, 2001), such as rolling supine in front of a potential partner (LeResche, 1976). Indeed, bonobos, engaging in locomotor-rotational movements, are highly likely to attract the attention of a partner, with play fighting ensuing (Palagi, 2008). In the vervets, over half of all these non-combat acrobatic movements led to subsequent playful contact. Indeed, the failures may be particularly instructive, as about $40 \%$ of these involved the performer standing up and attacking a nearby partner. For example, in one case, a monkey approached another and rolled onto the ground in front of its partner's face, about one body length away. After remaining like this for several seconds, its partner began to turn and walk away, at which time, the supine monkey stood up, circled in front of the other monkey and rolled onto the ground again. But after several seconds, when the standing monkey again began to move away, the supine monkey stood up and lunged at its partner and they engaged in a play fight. Thus, the majority of the non-combat related acrobatic maneuvers may be accounted for by the use of the movement as a self-handicapping tactic to solicit play from a potential partner.

The other $30 \%$, however, seemed to be performed for a different function. These were particularly evident in all those jumps that incorporated both roll and yaw in which the monkey flung itself so that its ventrum faced the flank of its partner. In these cases, the acrobatic maneuvers were coupled with failed attempts to grab the partner and so were linked to attack, but rather than facilitating the attack, it made the attack less likely to succeed. When this pattern of movement was used to facilitate attack, the pivot of the yaw was around the attacker's head. Its head remained fixed in space, opposed to its partner's shoulder, so enabling a bite to be delivered. In contrast, in the cases in which this maneuver did not facilitate contact, the pivot of the yaw was around the attacker's mid-body. In this case, its head and hands were moving through a large arc, thus reducing the chance of either a successful grab or bite. Such movements in these kinds of contexts are consistent with the training for the unexpected hypothesis (Špinka et al., 2001), in which the performer adds movements to its attack that undermines its own success (Pellis et al., 2005). Self handicapping that leads to loss of control may be critical to train the animal not to be flummoxed by unpredictable and uncontrollable situations (Pellis, Pellis, \& Bell, 2010). That is, the uses of these acrobatic maneuvers may be explicable by the training for the unexpected hypothesis.

The only hypothesis that did not seem useful in accounting for any of the acrobatic maneuvers observed was the motor training hypothesis (see also Nishida \& Inaba, 2009). The monkeys did not use jumps and rotations in a way that pushed their physical abilities to their limits as would be expected for improving physical ability (Simpson, 1976). Rather, most of the acrobatic maneuvers were performed in a 
way that was calibrated to the movements of one's partner's (combat adjustments) or were fairly standard, such as when rolling in front of a partner (as in solicitation). In a few cases, the acrobatic maneuvers that were performed in a manner consistent with training for the unexpected contained extremely exaggerated movements. These exaggerations seemed unnecessary for simple self-sabotage, and so could have been added to function for motor training as well. Even if some of these were performed for the function of motor training, this hypothesis would account for only a tiny fraction of all the acrobatic maneuvers performed. Nonetheless, even if these movements were not selected for their beneficial effects on motor training, the performance of these myriad jumps and rotations could have a beneficial influence on some aspects of motor performance as a byproduct. For example, in rats, play fighting in the juvenile period is critical for the development of social skills and emotional regulation (van den Berg et al., 1999) and affects the development of the executive areas of the brain (Baarendse, Counotte, O'Donnell, \& Vanderschuren, 2013; Bell, Pellis, \& Kolb, 2010), suggesting that such play functions to train high-level regulatory processes (Pellis et al., 2010). Yet such play is also correlated with changes in muscle structure and function and with changes to aspects of the motor system of the nervous system, suggesting a possible motor training benefit as well (Byers \& Walker, 1995).

\section{Conclusion}

Overall, this study found that, of the four hypotheses that have been proposed to account for acrobatic maneuvers during play fighting in vervet monkeys, the overwhelming majority could be accounted for by the combat hypothesis. That is, these maneuvers arise as a byproduct of attack and defense movements. When playful combat occurred in contexts in which such maneuvers were not useful for either attacking or defending, they were not used. Given that the other hypothesis did not link these maneuvers to their usefulness as combat tactics, if they were important for motor or psychological training or for communication purposes, then they should be used in all contexts, irrespective of whether in some contexts they would be muted. That this was not the case provides further strong evidence for the combat hypothesis. Once those maneuvers used for combat were excluded, this left an unexplained minority (about 18\%).

Most of this $18 \%$ were explicable in terms of them serving a communicatory function (i.e., soliciting play), and much of the rest were consistent with the maneuvers used for psychological training. Though highly skewed to the functional demands of combat, the production of acrobatic maneuvers during play fighting in vervet monkeys is multifunctional, raising the prospect that, across different species, different functions may be paramount. Not just the presence of such behavior needs to be measured, but detailed analyses of the form and context of the movements are needed to determine which hypotheses account for more behavior across different species. For example, sometimes, the mere presence of certain postures during play has been deemed sufficient to assume that they serve some function, such as communication (Sade, 1973). However, detailed analyses of the contexts of frequently performed postures show that only some are actually performed for communicatory functions (Yanagi \& Berman, 2014). The same analytical rigor is needed to discern the functions of acrobatic maneuvers during play.

\section{Acknowledgments}

We thank Linda Pellis with help in filming the Werribee troop and Nicola Forshaw and Miranda Lucas for help with the data collection from South Africa. We also thank Devin Cahoon for the drawings of the vervet monkeys and Heather Bell for help with the figures. The project was made possible by operating grants from the Natural Sciences and Engineering Research Council of Canada to LB, PH and SMP. 


\section{References}

Aldis, O. (1975). Play fighting. New York, NY: Academic Press.

Baarendse P. J., Counotte D. S., O'Donnell P., \& Vanderschuren, L. J. M. J. (2013). Early social experience is critical for the development of cognitive control and dopamine modulation of prefrontal cortex function. Neuropsychopharmacology, 38, 1485-1494.

Bell, H. C., Pellis, S. M., \& Kolb, B. (2010). Juvenile peer play experience and development of the orbitofrontal and medial prefrontal cortices. Behavioural Brain Research, 207, 7-13.

Bekoff, M. (2001). Social play behaviour: Cooperation, fairness, trust, and the evolution of morality. Journal of Consciousness Studies 8, 81-90.

Biben, M. (1998). Squirrel monkey play fighting: Making the case for a cognitive training function for play. In M. Bekoff \& J. A. Byers (Eds.), Animal play: Evolutionary, comparative, and ecological perspectives (pp. 161-2). Cambridge, UK: Cambridge University Press.

Blanchard, R. J., \& Blanchard, D. C. (1994). Environmental targets and sensorimotor systems in aggression and defense. In S. J. Cooper \& C. A. Hendrie (Eds.), Ethology and psychopharmacology (pp. 133-157). New York, NY: John Wiley \& Sons.

Blanchard, R. J., Blanchard, D. C., Takahashi, T., \& Kelley, M. J. (1977). Attack and defensive behaviour in the albino rat. Animal Behaviour, e, 622-634.

Brownlee, A. (1954). Play in domestic cattle in Britain: An analysis of its nature. British Veterinary Journal, e, 4868.

Byers, J. A., \& Walker, C. (1995). Refining the motor training hypothesis for the evolution of play. American Naturalist, 146, 25-41.

Eilam, D., \& Golani, I., (1988). The ontogeny of exploratory behavior in the house rat (Rattus norvegicus): The mobility gradient. Developmental Psychobiology, 21, 679-710.

Eshkol, N., \& Wachmann, A. (1958). Movement notation. London: Weidenfeld \& Nicholson.

Fagen, R. A. (1981). Animal play behavior. New York, NY: Oxford University Press.

Fontaine, R. P. (1994). Play as physical flexibility training in five ceboid primates. Journal of Comparative Psychology, 108, 203-212.

Foroud, A., \& Pellis, S. M. (2002). The development of 'anchoring' in the play fighting of rats: Evidence for an adaptive age-reversal in the juvenile phase. International Journal of Comparative Psychology, 15, 11-20.

Foroud, A., \& Pellis, S. M. (2003). The development of 'roughness' in the play fighting of rats: A Laban Movement Analysis perspective. Developmental Psychobiology, 42, 35-43.

Geist, V. (1978). On weapons, combat and ecology. In L. Krames, P. Pliner, \& T. Alloway (Eds.), Advances in the study of communication and affect, Vol. 4. Aggression, dominance and individual spacing (pp. 1-30). New York, NY: Plenum Press.

Golani, I. (1976). Homeostatic motor processes in mammalian interactions: A choreography of display. In P. P. G. Bateson \& P. H. Klopfer (Eds.), Perspectives in ethology, Vol. 2 (pp. 69-134). New York, NY: Plenum.

Hausfater, G. (1972). Intergroup behavior of free-living rhesus monkeys (Macaca mulatta). Folia Primatologica, $18,78-107$.

Heymer, A. (1977). Ethological dictionary. Berlin, Germany: Paul Parey.

Laidre, M. E. (2008). Do captive mandrills invent new gestures? Animal Cognition, 11, 179-187.

LeResche, L. A. (1976). Dyadic play in Hamadryas baboons. Behaviour, 57, 290-305.

Meaney, M. J., Stewart, J., \& Beatty, W. W. (1985). Sex differences in social play: The socialization of sex roles. Advances in the Study of Behavior, 15, 1-58.

Millar, S. (1981). Play. In D. McFarland (Ed.), The Oxford companion to animal behaviour (pp. 457-460). Oxford, UK: Oxford University Press.

Moran, G., Fentress, J. C., \& Golani, I. (1981). A description of relational patterns of movement during 'ritualized fighting' in wolves. Animal Behaviour, 29, 1146-1165.

Nishida, T., \& Inaba, A. (2009). Pirouettes: The rotational play of wild chimpanzees. Primates, 50, 333-341.

Owens, N. W. (1975a). Social play behaviour in free-living baboons, Papio anubis. Animal Behaviour, 23, 387-408.

Owens, N. W (1975b). A comparison of aggressive play and aggression in free-living baboons, Papio anubis. Animal Behaviour, 23, 757-765.

Palagi, E. (2008). Sharing the motivation to play: The use of signals in adult bonobos. Animal Behaviour, 75, 887896. 
Pasternak, G. M., Brown, L. R., Kienzle, S., Fuller, A., Barrett, L., \& Henzi, S. P. (2013). Population ecology of vervet monkeys in a high latitude, semi-arid riparian woodland. Koedoe, 55, 1-9. doi:10.4102/koedoe.v55i1.1078

Pellis, S. M. (1982). An analysis of courtship and mating in the Cape Barren goose Cereopsis novaehollandiae Latham based on Eshkol-Wachman Movement Notation. Bird Behaviour, 4, 30-41.

Pellis, S. M. (1988). Agonistic versus amicable targets of attack and defense: Consequences for the origin, function and descriptive classification of play-fighting. Aggressive Behavior, 14, 85-104.

Pellis, S. M. (1989). Fighting: The problem of selecting appropriate behavior patterns. In R. J. Blanchard, P. F. Brain, D. C. Blanchard, \& S. Parmigiani (Eds.), Ethoexperimental approaches to the study of behavior (pp. 361-374). Dordrecht, The Netherlands: Kluwer Academic Publishers.

Pellis, S. M. (1997). Targets and tactics: The analysis of moment-to-moment decision making in animal combat. Aggressive Behavior, 23, 107-129.

Pellis, S. M. (2011). Head and foot coordination in head scratching and food manipulation by purple swamp hens (Porphyrio porphyrio): Rules for minimizing the computational costs of combining movements from multiple parts of the body. International Journal of Comparative Psychology, 24, 255-271.

Pellis, S. M., \& Pellis, V. C. (1983). Locomotor-rotational movements in the ontogeny and play of the laboratory rat Rattus norvegicus. Developmental Psychobiology, 16, 269-286.

Pellis, S. M., \& Pellis, V. C. (1992). An analysis of the targets and tactics of conspecific attack and predatory attack in northern grasshopper mice Onychomys leucogaster. Aggressive Behavior, 18, 301-316.

Pellis, S. M., \& Pellis, V. C. (1997). Targets, tactics and the open mouth face during play fighting in three species of primates. Aggressive Behavior, 23, 41-57.

Pellis, S. M., \& Pellis, V. C. (1998). Structure-function interface in the analysis of play. In M. Bekoff \& J. A. Byers (Eds.), Animal play: Evolutionary, comparative, and ecological perspectives (pp. 115-140). Cambridge, UK: Cambridge University Press.

Pellis, S. M., \& Pellis, V. C. (2009). The playful brain. Venturing to the limits of neuroscience. Oxford, UK: Oneworld Press.

Pellis, S. M., Pellis, V. C., \& Bell, H. C. (2010). The function of play in the development of the social brain. American Journal of Play, 2, 278-296.

Pellis, S. M., Pellis, V. C., \& Foroud, A. (2005). Play fighting: Aggression, affiliation and the development of nuanced social skills. In R. Tremblay, W. W. Hartup, \& J. Archer (Eds.), Developmental origins of aggression (pp. 47-62). New York, NY: Guilford Press.

Pellis, S. M., Pellis, V. C., Pierce, J. D., Jr., \& Dewsbury, D A. (1992). Disentangling the contribution of the attacker from that of the defender in the differences in the intraspecific fighting of two species of voles. Aggressive Behavior, 18, 425-435.

Pellis, S. M., Pellis, V. C., Reinhart, R. J., \& Thierry, B. (2011). The use of the bared-teeth display during play fighting in Tonkean macaques (Macaca tonkeana): Sometimes it is all about oneself. Journal of Comparative Psychology, 125, 393-403.

Pellis, S. M., Blundell, M. A., Bell, H. C., Pellis, V. C., Krakauer, A. H., \& Patricelli, G. L. (2013). Drawn into the vortex: The facing-past encounter and combat in lekking male greater sage-grouse (Centrocercus urophasianus). Behaviour, 150, 1567-1599.

Petit, O., Bertrand, F., \& Thierry, B. (2008). Social play in crested and Japanese macaques: Testing the covariation hypothesis. Developmental Psychobiology, 50, 399-407.

Petrů, M., Špinka, M., Lhota, S., \& Sípek, P. (2008). Head rotations in the play of Hanuman langurs (Semnopithecus entellus): A description and an analysis of function. Journal of Comparative Psychology, 122, 9-18.

Petrů, M., Špinka, M., Charvátová, V., \& Lhota, S. (2009). Revisiting play elements and self-handicapping in play: A comparative ethogram of five Old World Monkey species. Journal of Comparative Psychology, 123 , 250-263.

Reinhart, C. J., Pellis, V. C., Thierry, B., Gauthier, C.-A., VanderLaan, D. P., Vasey, P. L., \& Pellis, S. M. (2010). Targets and tactics of play fighting: Competitive versus cooperative styles of play in Japanese and Tonkean macaques. International Journal of Comparative Psychology, 23, 166-200.

Ruehlmann, T. E., Bernstein, I. S., Gordon, T. P., \& Balcaen, P. (1988). Wounding patterns in three species of captive macaques. American Journal of Primatology, 14, 125-134.

Sade, D. S. (1973). An ethogram of rhesus monkeys: I. Antithetical contrasts in posture and movement. American Journal of Physical Anthropology, 38, 537-542.

Schaller, G. B. (1963). The mountain gorilla: Ecology and behavior. Chicago, IL: The University of Chicago Press.

Siegel, S., \& Castellan, N. J. J. (1988). Nonparametric statistics for the behavioral sciences. New York, NY: 


\section{McGraw Hill.}

Simpson, M. J. A. (1976). The study of animal play. In P. P. G. Bateson \& R. A. Hinde (Eds.), Growing points in ethology (pp. 385-400). Cambridge, UK: Cambridge University Press.

Smith, P. K. (1997). Play fighting and real fighting: Perspectives on their relationship. In A. Schmitt, A. Atswanger, K. Grammar, \& K. Schafer (Eds.), New aspects of human ethology (pp. 47-64). New York, NY: Plenum Press.

Špinka, M., Newberry, R. C., \& Bekoff, M. (2001). Mammalian play: Can training for the unexpected be fun? Quarterly Review of Biology, 76, 141-176.

Symons, D. (1978). Play and aggression. A study of rhesus monkeys. New York, NY: Columbia University Press.

Thierry, B., Aureli, F., Nunn, C. L., Petit, O., Abegg, C., \& de Waal, F. B. M. (2008). A comparative study of conflict resolution in macaques: Insights into the nature of trait covariation. Animal Behaviour, 75, 847860.

de Waal, F. B. M., \& Luttrell, L. M. (1989). Toward a comparative socioecology of the genus Macaca: Different dominance styles in rhesus and stumptail monkeys. American Journal of Primatology, 19, 83-109.

van den Berg, C. L., Hol, T., van Ree, J. M., Spruijt, B. M., Everts, H., \& Koolhaas, J. M. (1999). Play is indispensable for an adequate development of coping with social challenges in the rat. Developmental Psychobiology, 34, 129-138.

Whitten, P. L., \& Smith, E. O. (1984). Patterns of wounding in stumptail macaques. Primates, 25, 326-336.

Yanagi, A., \& Berman, C. M. (2014). Body signals during social play in free-ranging Rhesus macaques (Macaca mulatta): A systematic analysis. American Journal of Primatology, 76, 168-179. 